\title{
Title
}

Moderators of the Effect of Psychological Interventions on Depression and Anxiety in Cardiac Surgery Patients: A Systematic Review and Meta-analysis.

\section{Full citation:}

Protogerou, C., Fleeman, N., Dwan, K., Richardson, M., Dundar, Y., \& Hagger, M. S. (2015). Moderators of the effect of psychological interventions on depression and anxiety in cardiac surgery patients: A systematic review and meta-analysis. Behaviour Research and Therapy, 73, 151-164. doi: 10.1016/j.brat.2015.08.004

\section{Acknowledgment}

We thank Professor Blair T. Johnson, University of Connecticut and Dr Angela Boland, University of Liverpool, for their input on a prior draft of this article. 


\begin{abstract}
Cardiac surgery patients may be provided with psychological interventions to counteract depression and anxiety associated with surgical procedures. This systematic review and metaanalysis investigated whether intervention efficacy was impacted by type of cardiac procedure/ cardiac event; control condition content; intervention duration; intervention timing; facilitator type; and risk of bias. MEDLINE, EMBASE, and PsycINFO were searched for randomized controlled trials comparing anxiety and depression outcomes, pre and post psychological and cardiac interventions. Twenty-four studies met the inclusion criteria for the systematic review $(N=2718)$ and 16 of those were meta-analyzed $(N=1928)$. Depression and anxiety outcomes were reduced more in interventions that lasted longer, were delivered postsurgery, and by trained health professionals. Depression (but not anxiety) was reduced more when the experimental intervention was compared to an 'alternative' intervention, and when the intervention was delivered to coronary artery bypass graft patients. Anxiety (but not depression) was decreased more when interventions were delivered to implantable cardioverter defibrillator patients, and were of 'high' or 'unclear' risk of bias. In addition to estimating efficacy, future work in this domain needs to take into account the moderating effects of intervention, sample, and study characteristics.
\end{abstract}

Keywords: depression and anxiety; cardiac surgery patients; psychological interventions; evidence synthesis; moderator effects. 
Coronary heart disease (CHD) is the leading cause of morbidity and death, globally (Hoyert \& Xu, 2012; WHO, 2011). CHD treatment varies from taking medication and modifying behavior, to invasive cardiac procedures that usually include catheterisation, implantation of battery-operated devices, and open-heart surgery. Overall, the literature suggests that invasive cardiac procedures improve patient physical health and functioning. As a consequence, research has focused on evaluating patients' psychological well-being (Ai, Park, Huang, Rodgers, \& Tice, 2007; Denollet, Schiffer, \& Spek, 2010; Pedersen \& Denollet, 2006; Škodová et al., 2009). While the literature suggests that cardiac surgery patients experience better psychological well-being post-surgery (Höfer et al., 2005; Shephard \& Franklin, 2001), a substantial subgroup of these patients (approximately $20 \%$ to $30 \%$ ) report a deterioration of physical functioning and increased psychological distress (Hawkes \& Mortensen, 2006; Škodová et al., 2009).

Patients who have undergone, or, are about to undergo, invasive cardiac procedures have been shown to be prone to high levels of distress. For example, up to $87 \%$ of implantable cardioverter defibrillator (ICD) patients may experience some degree of anxiety, while up to $38 \%$ of those patients may experience symptoms compatible to anxiety disorder (Bostwick \& Sola, 2007). In addition, 15-20\% of myocardial infarction (MI) patients experience symptoms of major depression (Hanssen, Nordrehaug, Eide, Bjelland, \& Rokne, 2009; Thombs et al., 2006). In order to counteract depression and anxiety associated with cardiac procedures, cardiac patients may be provided with psychological interventions. Previous meta-analyses have investigated the efficacy of such interventions in reducing postoperative anxiety and depression in cardiac patients, and have yielded inconclusive results. For example, (Dusseldorp, van Elderen, Maes, Meulman, \& Kraaij, 1999) found no benefit of 'psycho-educational' programmes on patient anxiety and depression, whereas (Whalley, Thompson, \& Taylor, 2014) found significant benefits. Inconsistent results across meta- 
analyses may be due, in part, to variability in study foci, outcome variables, and patient population included, making generalizations of findings difficult. For instance, (van Dixhoorn \& White, 2005) included only myocardial ischaemia patients, while (Whalley et al., 2014) excluded ICD patients and (Linden, Phillips, \& Leclerc, 2007) primarily focussed on mortality and morbidity outcomes. An additional limitation of existing meta-analyses is the lack of subgroup analyses (moderator effects), even though the included psychological interventions are heterogeneous (Whalley et al., 2011). Concerns have also been raised (Thompson \& Ski, 2013) as to what constitutes a 'psychological' intervention. This is an important concern given that some previous meta-analyses (Rees, Bennett, West, Davey, \& Ebrahim, 2004; Welton, Caldwell, Adamopoulos, \& Vedhara, 2009) have not made distinctions between psychological and non-psychological (e.g., physiotherapy, exercise, massage) components, making it thus difficult to isolate benefits solely attributable to the psychological components (Whalley et al., 2014). A clear understanding of intervention effects is more likely to be accomplished by isolating specific parameters impacting outcomes, which can reflect the possible underlying mechanisms through which effects are obtained (Michie, 2008).

This systematic review and meta-analysis aimed to add to the existing literature on the effectiveness of psychological interventions to reduce distress in cardiac patients and resolve some of the inconsistencies observed in previous meta-analytic syntheses of these data. Specifically, the current analysis aimed to assess the efficacy of psychological interventions to reduce anxiety and depression in patients undergoing cardiac procedures. We also aimed to identify the moderating factors (e.g., risk of bias, intervention duration, timing of the intervention, type of control group, delivery method) that diminish or magnify the effects of interventions on distress reduction in cardiac patients. A limitation of previous meta-analyses 
is the lack of a systematic test of moderators and such an analysis may account for the inconsistencies in the observed effect sizes across previous reviews.

\section{Methods}

\section{Clarification of Constructs}

An important initial step in identifying the impact of psychological interventions on cardiac patients' distress was to adopt accepted criteria for the definition and operationalization of psychological interventions. In the current analyses, interventions had to be based on identifiable psychological theories or psychological techniques stemming from those theories (e.g., socio-cognitive theory, learning theory, psychodynamic). This inclusion criterion was adopted to ensure a level of quality control over the interventions in the studies included in the current analyses. We also stipulated that interventions were not to be combined with non-psychological (e.g., physiotherapy, massage, exercise) components likely to confound the effects of the psychological interventions. We use the term 'experimental interventions' to refer to the target psychological interventions that were tested against a control condition, often 'usual care'. A small number of studies compared the experimental intervention against an alternative psychological intervention, instead of, or in addition to, a control condition. We use the term 'alternative interventions' to refer to the latter. We use the term 'distress' as a collective term for anxiety and depression (Mirowsky \& Ross, 2002). We use the term 'moderators' to refer to intervention, study, and sample features, that were expected to affect the direction and/or strength of effect size estimates. Our meta-analysis focussed specifically on depression and anxiety outcomes, as measured by validated scales.

\section{Eligibility Criteria}

To be included, studies had to be randomised controlled trials (RCTs) that: (1) assessed the efficacy of a psychological intervention, as defined above; (2) were published from 1980 onwards; (3) included individuals aged 18 years or older, having undergone or 
were about to undergo an invasive cardiac procedure; (4) included measures comparing pre and post intervention depression and anxiety by means of validated scales; (5) were published in the English language; and (6) were published full-text. Studies were excluded if they: (1) included 'psychological' interventions that deviated from the above definition; (2) psychological interventions aiming to modify outcomes other than psychological distress (e.g., morbidity, mortality, adherence to medication, exercise, bodily symptoms); (3) were duplicates of another RCT; (4) were abstract-only reports; and (5) did not measure depression and anxiety by means of a validated scale. We focus exclusively on RCTs as this design is considered to be the 'gold standard' used to establish the efficacy of health-related interventions (Norman \& Streiner, 1993). The year 1980 was chosen as the earliest date for studies since the first ICD transplantation took place then, and rehabilitation programmes comprising psychological components for this patient group were subsequently developed. We included studies of patients who had undergone, or were about to undergo, a cardiac procedure as we wanted to assess whether the timing of the intervention, relative to the cardiac procedure, would impact anxiety and depressions outcomes. Studies measured depression and anxiety pre and post psychological and cardiac intervention. Inclusion was restricted to studies utilizing validated to enhance accuracy and comparability of findings.

\section{Search Strategy}

We conducted an exhaustive search of electronic databases including MEDLINE, MEDLINE In-Process and Other Non-Indexed Citations, PsycINFO and EMBASE for the period from 1980 to July 2013. We also searched the reference lists of identified studies and Google Scholar. Search terms for electronic databases included a combination of index terms (e.g., types of cardiac and vascular invasive surgical procedures) and free text words (e.g., psychological interventions) combined with specific conditions (e.g., depression, anxiety, emotional or psychological distress). A number of authors were contacted, via email, in order 
to obtain additional information not reported in the published RCTs. An updated search was conducted in March 30, 2015 using the same search terms and databases, yielding four additional studies. Twenty-four RCTs met the inclusion criteria for the systematic review and 16 of those provided data suitable for the meta-analysis. Study selection and reasons for exclusion are presented in a flow chart (figure 1) based on PRISMA guidelines (Moher, Liberati, Tetzlaff, \& Altman, 2009). Two independent coders screened the abstracts for eligibility (stage 1 inclusion), then the full copies of eligible titles were independently screened using a priori inclusion-exclusion criteria, and then, the final list of included studies was identified (stage 2 inclusion). Disagreements about study inclusions were resolved by discussion and by consulting with a third coder. There were no geographical or publication outlet restrictions. The results of a complete search strategy are available online.

\section{Data Extraction}

A coding form was developed specifically for this meta-analysis, based on recommendations by (Lipsey \& Wilson, 2001). The coding form captured: (a) study level descriptors (e.g., publication year, type, and location); (b) study sample descriptors (e.g., sample size, age, gender, type of cardiac procedure undertaken); (c) experimental and alternative intervention descriptors (e.g., duration, setting, medium, facilitator type); (d) effect size level descriptors (e.g., outcome category, scales used, means, medians, standard deviations, sample sizes at appropriate measurement times); and (e) risk of bias. The coding form was independently pilot-tested by two coders (CP, NF) using $25 \%$ of the eligible studies, and inter-coder disagreements were resolved through discussion. All eligible studies were then coded independently by two coders and once again, disagreement was resolved through discussion.

\section{Data Preparation and Analyses}


Change from baseline in depression and anxiety was the primary outcome variable. The standardized mean difference (Hedges' $g$; Hedges \& Olkin, 1985) was the chosen effect size metric for the intervention effect as different scales were used within the studies. Change from baseline difference was within-groups (i.e., the same distress outcome measures were obtained before and after the intervention for all groups), but the differences reported were between-groups (i.e., comparisons were made for intervention versus control groups). The 95\% confidence intervals of the effect size were also computed. Where the studies did not report the standard deviation (SD) for change from baseline, this was calculated according to accepted guidelines (Higgins \& Green, 2008, p. 488). To illustrate, the values $r=.50$ and $r=$ .70 represent the range reported in one of the studies (Sorlie, Busund, Sexton, Sexton, \& Sorlie, 2007). In this instance, the middle value of $r=.60$ was chosen for primary analyses, and sensitivity analyses (i.e., exploration of whether main findings change by varying aggregation method) were conducted using the upper and lower bounds of the correlation coefficient. This was to ensure that the selection of the center value was appropriate. Outcome measures were summarized at post-intervention (earliest measurement taken after the psychological intervention) and follow-up (earliest measurement taken three months or more after the psychological intervention). The included RCTs compared at least two of the following conditions: experimental intervention, alternative intervention, and usual care control. Thus, outcomes were separately compared between the experimental intervention and usual care control conditions, as well as the experimental and alternative intervention conditions. Heterogeneity was assessed with the $I^{2}$ index, which offers the percentage of the variability in effect estimates due to heterogeneity rather than sampling error. $I^{2}$ values of $.25), .50$, and .75 translate to low, moderate, and high levels of heterogeneity, respectively (Higgins \& Thompson, 2002). Risk of bias (i.e., threat to internal validity) was assessed using the Cochrane Collaboration's risk of bias tool (Higgins \& Green, 2008). The risk of bias tool 
evaluates selection bias, performance bias, withdrawal/attrition bias, detection bias, and reporting bias. Due to the nature of the interventions, assessing blinding of treatment assignment was not appropriate. In addition to assessing risk of bias separate domains, we created an overall (un-weighted) risk of bias score, by assigning to each domain a score of 1 for low risk of bias, 2 for unclear risk of bias, and 3 for high risk of bias, and summing these. An 'overall low' risk of bias estimation was given to studies that scored $\leq 6$; an 'overall unclear' risk of bias estimation was given to studies that scored between 7-12; and an 'overall high' risk of bias estimation was given to studies that scored $\geq 12$. Two reviewers assessed all risk of bias studies independently and discrepancies were resolved through discussion.

Possible asymmetries in the distribution of effect sizes against study precision, as an indicator of small-study bias, were analyzed with the Egger et al.'s (1997) test (Egger, Davey Smith, Schneider, \& Minder, 1997) and Begg and Mazundar's (1994) strategy (Begg \& Mazundar, 1994). A random effects model of meta-analysis was used because simulation data using this model suggest that it will provide the most robust estimates under conditions of high heterogeneity (DerSimonian \& Laird, 1986). Studies were grouped and analysed separately for each moderator, assessing the impact of the following six moderators on the intervention effect: (1) type of cardiac procedure/cardiac event (CABG, ICD, other); (2) control condition content (usual medical care only; usual medical care plus additional content, typically psycho-education; usual care including a brief form of the experimental intervention; other); (3) intervention duration (short/up to one week, medium/up to six weeks, long/over six weeks, not reported); (4) facilitator type (trained psychologist, other trained health professional, student); (5) timing of psychological intervention (before or after the cardiac procedure); and (6) overall risk of bias. These features were chosen as authors of previous studies have identified them as potential moderators of the psychological intervention-distress reduction relationship (Sears et al., 2007). While an examination of the simultaneous effect of 
these moderator variables on study outcomes is desirable, the small numbers of studies in each moderator group precluded a multivariate analysis. All data analyses, including risk of bias assessment and figures, were carried out with Review Manager (RevMan, version 5.2) software.

\section{Results}

\section{Description of Studies}

Studies sampled 2718 cardiac patients who were predominately male (79\% of studies), and with mean ages between 53.30 and 68.70 for the intervention groups, 54.40 and 68.0 for the control groups, and 57.65 and 64.30 for the alternative intervention groups. Over half of the studies were conducted in the United States $(k=14,58 \%)$. Sample sizes varied from 15 to 289 . Nine (45\%) studies included only CABG patients, eight studies (33\%) included only ICD patients, and seven studies (29\%) included patients who had had one or another type of cardiac procedure/event. Fourteen interventions (58\%) were delivered via, or, assisted by, technology (i.e., audiotape, video tape, compact disc/computer, telephone, and the internet). Seventeen studies (71\%) included a usual care-only control condition as a comparator, while three (12\%) studies provided only an alternative intervention as comparator, and four (16\%) studies offered an alternative intervention in addition to the control. Fourteen interventions (58\%) could be characterized as 'long term', as they were delivered for a minimum of six weeks, six interventions (25\%) lasted up to 6 weeks ('medium term'), and two interventions (.8\%) lasted up to one week ('short term'). We were unable to ascertain the length of two interventions (.8\%), despite contacting authors. Most interventions $(k=19,79 \%)$ were delivered post-surgery. All interventions, regardless of their timing, stated that they aimed to reduce post-surgery distress. Three of the pre-surgery interventions also aimed to reduce non-psychological outcomes, i.e., reducing the length of hospital stay and service utilization. Thus, we found that the main difference between pre- 
and post- surgery interventions was that some pre-surgery interventions also aimed to assess cost utility of the intervention. Maximum follow-up periods varied, from one week to two years. Ten $(42 \%)$ studies used a six-month follow-up measure. Most interventions were delivered at a hospital setting (even if there were additional sessions at the patient's home) $(k=16,67 \%)$. Nine (37\%) interventions were delivered by nurses, eight $(33 \%)$ were delivered by psychologists, one was delivered by a trained peer volunteer $(.4 \%)$, one $(.4 \%)$ was delivered by a 'trained health professional', and four were self-delivered (16\%). Depression was mostly measured by the Beck Depression Inventory (BDI) (Beck, Ward, Mendelson, Mock, \& Erbaugh, 1961) and the Hospital Anxiety and Depression Scale (HADS) (Zigmond \& Snaith, 1983), in nine (37\%), and six studies (25\%) respectively. Anxiety was mostly measured using the State-Trait Anxiety Inventory (STAI) (Speilberger, Gorsuch, Lushene, Vagg, \& Jacobs, 1983) and the HADS, in seven (26\%) and six (25\%) studies, respectively. Finally, most interventions $(k=18,75 \%)$ were based on cognitive-behavior or social learning theory (Bandura, 1977), utilizing techniques, such as identifying and reframing negative thoughts; identifying and dealing with stressful situations; coping strategies; setting personal goals and ways of achieving those goals; group discussions with emphasis on group support; guided imagery; and stress reduction - relaxation techniques. While five additional interventions (21\%) were labelled as types of counselling, support, or stress management programmes, they too incorporated cognitive behaviour techniques. Only one intervention (.4\%) indicated using techniques drawn from existential psychological theory. Thus, based on our coding, we concluded that all but one psychological intervention were based on cognitive-behavior theory principles and techniques. Table 1 provides a summary of included study characteristics and findings. Brief descriptions of the included interventions and control or comparator conditions are available as online supplemental material.

\section{Risk of Bias in Included RCTs}


For more than half of the included studies $(k=14,58 \%)$ overall risk of bias was unclear. For three studies (12.5\%) overall risk of bias was deemed low and for seven studies (29\%) risk of bias was assessed to be high. The kappa statistic for the overall risk of bias was 0.72 (95\% CI: 0.43, 1.00), indicating substantial agreement between the two assessors. Most studies clearly reported randomization procedures reflecting adequate random sequence generation $(k=19,79 \%)$, whereas the remaining studies did not report full details of randomization procedures. Allocation concealment was unclear for half studies $(k=12$, $50 \%$ ), with ten studies ( $41 \%$ ) clearly reporting the method used to conceal the allocation sequence. Only seven studies (29\%) reported that outcome assessors were blinded to group allocation. About thirteen studies (54\%) indicated that data were either not missing or that missing data were handled adequately (e.g., used intention-to-treat analyses); eight of these studies $(33 \%)$ used intention-to-treat analyses to deal with attrition. Half of the studies $(k=$ $12,50 \%$ ) reported outcomes completely and accurately (e.g., studies presented pre-specified outcomes, reported in full detail). Thus, the strongest methodological areas of included RCTs related to randomization procedures and data reporting (i.e., low selection and reporting bias), while the weakest methodological area related to blinding of outcome assessment (i.e., unclear-high detection bias). Figures 2 and 3 depict authors' risk of bias assessment.

\section{Quantitative Analyses}

We tested out main hypotheses by applying random-effects meta-analysis to data on the effect of psychological interventions on post-surgery indices of distress, i.e., depression and anxiety across the sample of studies.

Change in depression and anxiety. Fifteen studies reporting data for depression and twelve studies reporting data for anxiety $(N=1928)$ were meta-analyzed. Relative to controls, experimental interventions succeeded in reducing depression at post-intervention ( $g$ $=-0.84,95 \%$ CI: -1.30 to $-0.38, k=16)$ and at follow-up $(g=-0.72,95 \%$ CI: -1.30 to -0.13 , 
$k=9)$. Similarly, interventions succeeded in reducing anxiety at post-intervention $(g=-0.62$, 95\% CI: -1.04 to $-0.21, k=12)$ and at follow-up ( $g=-0.64,95 \%$ CI: -1.22 to $-0.07, k=7)$. Relative to alternative interventions, experimental interventions did not significantly reduce depression or anxiety at post-intervention [( $g=-0.17,95 \% \mathrm{CI}:-1.09$ to $0.76, k=5)$ and $(g=$ $0.17,95 \% \mathrm{CI}:-0.13$ to $0.47, k=4)$, respectively]; or at follow-up [( $g=-0.17,95 \% \mathrm{CI}:-1.07$ to $0.74, k=4)$ and ( $g=0.78,95 \% \mathrm{CI}:-0.86$ to $2.42, k=3)$, respectively]. Heterogeneity was high $\left(I^{2}>.75\right)$, suggesting that results varied more across studies than expected by sampling error alone, and that more complex analyses (i.e., moderator analyses) were indeed warranted. Depression and anxiety outcomes were still significantly reduced at both time points, after varying the correlation coefficient that was used in the calculation of the SD for change from baseline (i.e., sensitivity analysis). One study (Freedland et al., 2009) indicated a much larger intervention effect than the remaining studies (i.e., > 3 standard deviations away from the mean). We treated this study as an outlier in our sensitivity analysis to examine whether the effects of the intervention would be altered with the exclusion of a particularly large (outlying) effect size. The removal of this study resulted in a substantial attenuation of the estimates of the intervention effect, but the intervention still led to a statistically significant reduction in depression at post-intervention ( $g=-.49,95 \%$ CI: -.85 to $-.13, k=15)$ and anxiety at post-intervention $(g=-.36,95 \% \mathrm{CI}$ : -.62 to $.09, k=11)$, and anxiety at followup ( $g=-.24,95 \%$ CI: -.41 to $-.07, k=6)$. Forest plots generated by meta-analyses assessing changes in depression and anxiety (experimental versus control conditions) are presented in figures 4-11. The remaining forest plots - including sensitivity analyses - are available as online supplemental material. There were asymmetries in the distribution of the effect sizes against study precision using regression techniques indicating the presence of small-study bias (Egger et al.'s (1997) test $[(t=-2.18, p=.04) ;(t=-3.02, p=.01)]$; Begg's (1994) test $[z$ 
$=-2.92, p=.004 ; z=-2.88, p=.004)$ for depression and anxiety, respectively. Funnel plots of effect sizes are available as online supplemental material.

Moderators of change in depression and anxiety. Post-intervention depression decreased more when the experimental intervention (a) lasted longer, i.e., over six weeks $(z=$ $2.70, p=.007) ;(\mathrm{b})$ was delivered post-surgery $(z=3.20, p=.001)$; (c) was compared to an intervention that offered 'education and advice' about surgery and aftercare $(z=12.37, p<$ $.00001)$; (d) was delivered by a trained psychologist $(z=2.75, p=.006)$. Furthermore, depression at this time point decreased more for CABG patients $(z=2.97, p=.003)$. Depression at follow-up decreased more when the experimental intervention was delivered post-surgery $(z=2.53, p=.01)$. Furthermore, depression at this time point was decreased more in two interventions that did not state 'duration' $(z=4.49, p=.0001)$, and for CABG patients $(z=2.26, p=.02)$. Anxiety (post-intervention; follow-up) was reduced more when the experimental condition: (a) lasted longer/over six weeks $[(z=2.10, p=.04) ;(z=3.13, p$ $=.002)]$; (b) was delivered after the cardiac procedure $[(z=2.45, p=.01) ;(z=2.05, p=$ .01)]. Anxiety (post-intervention only) was reduced more when the experimental condition was (a) compared to a usual care condition $(z=2.72, p=.007)$; (b) delivered by a trained psychologist $(z=2.36, p=.02)$; (c) risk of bias was deemed high $(z=3.56, p=.0004)$. Anxiety (follow-up only) decreased more when the experimental condition was (a) delivered by a trained health professional $(z=2.70, p=.007)$; and (b) risk of bias was 'unclear' $(z=$ $3.05, p=002)$. Finally, anxiety at this time point decreased more for ICD patients $(\mathrm{z}=2.86, p$ $=.004)^{1}$. We have included as online supplemental material forest plots generated by moderator analyses, as well as a table of subgroup effect sizes and related statistics of depression and anxiety reduction at post-intervention and follow-up.

\footnotetext{
${ }^{1}$ It is important to note that all of the moderator analyses were conducted separately and do not reflect multivariate effects (e.g., meta-regression) of the moderators on the outcome variables. The small numbers of studies in each moderator group precluded a meta-regression examining the simultaneous effects of moderator variables on study outcomes.
} 


\section{Discussion}

The current meta-analysis aimed to examine the efficacy of psychological interventions to attenuate anxiety and depressive responses in cardiac surgery patients. Our analysis is the first to examine the impact of specific study, sample, and intervention features as moderators of the effect of psychological interventions on distress in cardiac surgery patients. Our meta-analysis provides preliminary evidence that the tested interventions in the current sample of studies significantly decreased depression and anxiety relative to controls.

Our findings indicated that changes in these outcomes were medium to large in size and were sustained for a minimum of three months. These findings are consistent with previous research that have shown psychological interventions to be effective in reducing anxiety and depression outcomes in cardiac surgery patients (Whalley et al., 2014), although we also note that our current finding also exhibit the substantive heterogeneity in effects previously identified. In conducting an evaluation of candidate moderator variables, we aimed to systematically resolve this between-study variability.

Our univariate moderator analyses indicated that interventions that ameliorated both depression and anxiety lasted longer, were delivered after the cardiac procedure, and were compared with some type of intervention (i.e., interventions adopting education and counselling techniques). It therefore seems that intervention techniques can be more effective when delivered for at least six weeks, as compared to shorter times. It also appears that psychological interventions may have more of an impact when delivered post-cardiac surgery. The moderating effect of control condition content implies that providing usual care only, even if that comprises education and counselling, may not be enough to reduce distress. Current data therefore suggest that a separate psychological intervention can be a beneficial addition to usual care. The type of cardiac procedure undertaken appeared to influence depression and anxiety outcomes. CABG patients reported greater depression reduction than 
patients undergoing ICD and other procedures, while ICD patients reported greater anxiety reduction. There is evidence to suggest that, compared to anxiety, depression is more prevalent in CABG patients (Tully \& Baker, 2012). ICD patients appear to be more prone to anxiety, with some evidence to suggest that ICD procedures may induce anxiety disorders, de novo (Sola \& Bostwick, 2005). It is possible, therefore, that the interventions included in this meta-analysis were most effective for CABG patients who tend to suffer more depression. Facilitator type influenced depression and anxiety outcomes. Depression and anxiety at postintervention was reduced most when delivered by trained psychologists, but anxiety at follow-up was reduced when delivered by 'other trained health professionals', especially nurses. Thus, it appears that health professionals coming from different academic backgrounds can be equally effective in delivering these types of interventions for particular outcomes, assuming that they have the necessary training. Finally, overall risk of bias only affected anxiety outcomes. Anxiety, at both time points, was reduced more in interventions of unclear or high risk of bias.

\section{Study Strengths and Limitations}

The current meta-analysis is the first to look at specific moderator variables of the effect of interventions on distress in cardiac surgery patients that have not been accounted for in previous meta-analyses and systematic reviews, such as intervention timing. Precision and accuracy of results was enhanced given that studies were included if they delivered psychological content; obtained anxiety and depression measures via robust, validated scales; and offered enough information to explore moderator effects. Moreover, outcome measures were summarized across time, at baseline, earliest post intervention, and a minimum of three months' follow-up. Subsequent to the two types of sensitivity analyses, a significant intervention effect was obtained for depression and anxiety at post-intervention and followup. A further strength of the current analysis is the adoption of rigorous study search, 
identification, and classification procedures. Specifically, study search and was carried out by an information specialist (YD), data extraction and coding were conducted by experienced reviewers $(\mathrm{CP}, \mathrm{NF})$, and authors were contacted to obtain additional information. Using experienced searchers and coders, and adding a supplemental search component, substantially enhances reporting quality (Mullins, DeLuca, Crepaz, \& Lyles, 2014). In addition, a risk of bias assessment of included RCTs was conducted, highlighting areas of methodological strength and weakness.

As is the case with all meta-analyses, our meta-analysis mirrors limitations of the included primary studies. Detail about intervention content was sometimes minimal in the RCTs, often without specifying which particular techniques and strategies were used or linked to better outcomes. Thus, although our findings suggest that psychological interventions guided by cognitive behavior theory do work, it was not possible to ascertain which techniques and strategies work best. Similarly, the content of usual care comparison groups tended to be inadequately reported or was not always neutral. For example, in some cases, 'usual care' still meant that patients were exposed to some kind of treatment resembling the active intervention content. The 'right' type of control group is imperative in psychological interventions, as content of control condition can affect the effect size of the active intervention (Lindquist, Wyman, Talley, Findorff, \& Gross, 2007). Detailed demographic information was often lacking in the included studies and most studies were conducted in countries in the US, with predominately male participants. Limited demographic detail precluded us from conducting moderation analyses with demographic variables, or generalizing to other countries or cultures.

In addition, despite extensive search of studies, only a relatively a small number of RCTs met our inclusion criteria and were included in the meta-analyses $(k=16)$. Our analyses also revealed high levels of heterogeneity across studies in the effects of the 
intervention on depression and anxiety outcomes, a possible reflection of the multiple generative mechanisms underlying the 'high distress - poor cardiac outcomes' relationship (Whalley et al., 2011). Furthermore, the small number of studies meant that the sample sizes for moderator groups were also small, indicating that such analyses should be treated as preliminary and exploratory. Given the small sample sizes, there is, of course, the potential for relatively few effects from larger samples to affect the effect sizes, particularly in the moderator groups with smaller samples sizes. We view tests of these moderators as a first step in attempting to resolve heterogeneity of these interventions in cardiac surgery patients. It is important to note that the tests of small-study bias are heavily influenced by study heterogeneity, such that no definitive conclusions can be drawn regarding the source of bias in such tests and the findings may be unreliable in the presence of high levels of heterogeneity (Hagger \& Chatzisarantis, 2014; Ioannidis \& Trikalinos, 2007; Peters et al., 2010). As with all studies where there may be multiple sources of small-study bias, we cannot definitively conclude the source of the bias identified in the current analysis. Disentangling the different sources of bias may require, for example, the conduct of metaregression analyses predicting effect sizes by discrete and continuous covariates on which the effect size may depend and comparing the findings alongside tests of small-study bias as recommended by Peters et al. (2010). We look to future research to continue to update these findings as the numbers of studies in the field increases and makes sufficient data to test effects of candidate moderators of the effect available. We also hope that our analysis may provide some guidelines for future research that will ramp up the quality of studies, a shift that may also contribute to a better resolution of the heterogeneity in the intervention effects observed in the current sample of studies.

In addition, our analysis revealed only three studies with low risk of bias, indicating unclear or poor methodological quality across studies. Our moderator analyses indicated that 
risk of bias impacted anxiety outcomes only, with most of those studies being of 'unclear' risk of bias; therefore this outcome could be attributed to poor study reporting rather than poor design. This notwithstanding, it has been pointed out that the Cochrane risk of bias tool criteria maybe overly strict (Hempel et al., 2013), probably because it was initially meant to assess non-behavioural randomized interventions.

\section{Insights for Practice and Research}

A key finding of the current meta-analysis is that the effect of psychological interventions on anxiety and depression outcomes in cardiac surgery patients is independently moderated by each of the following factors:(a) delivering psychological content for longer time periods - at least for six weeks; (b) considering patient characteristics relating to the cardiac procedure undertaken and tailor content appropriately; (c) delivering the intervention post-surgery; (d) limiting methodological biases; and (e) using trained health professionals as facilitators. As reflected in our findings, each of these five factors serve as sources of potential heterogeneity in the RCTs of this research area, and it is thus recommended that future trials are calibrated accordingly. Apropos, heterogeneity is bound to be associated with methodological biases within and across RCTs and it is clear that future trials need to eliminate such biases. Published guidelines exist regarding ways of enhancing RCT internal and external validity, such as the CONSORT Statement (Egger, Jüni, \& Bartlett, 2001; Moher, Schulz, \& Altman, 2001) and the "Mind the Gap" mnemonic for designing and reporting randomized trials (Hartling et al., 2012), but none of the RCTs we came across indicated adhering to any known schemes. The between-study heterogeneity is also linked to characteristics of the intervention recipients (Horodyska et al., 2015) and, in relation to this, our findings indicated that intervention efficacy differed as a function of cardiac procedure. Consequently, psychological research needs to further clarify how person-centred patient variables (e.g., type of cardiac condition and procedure undertaken, personality 
characteristics, trait affect) interact with cardiac outcomes and consider controlling for their impact. Furthermore, the results suggest that psychological interventions can be more effective than usual care in reducing depression and anxiety in cardiac surgery patients, even when usual care comprises education, counselling, or a brief form of the intervention. Thus, it seems that is worth investing in developing separate distress reduction interventions for this patient population. Finally, while cognitive behavior techniques appeared to be successful in ameliorating depression and anxiety, clearer descriptions of intervention content and delivery is needed. As interventions will utilize multiple techniques, it is important to know which techniques are the more effective. Knowledge of the effectiveness of specific techniques is important as it relates to the effectiveness, efficiency, and feasibility of distress-reduction interventions for cardiac patients (Salmoirago-Blotcher \& Ockene, 2009). Given that multicomponent interventions are more expensive and more challenging to deliver, the inclusion of ineffective components would unnecessarily ramp-up costs for little or no gain with respect to health outcomes. 


\section{References}

(*Asterisked citations are the included RCTs.)

Ai, A. L., Park, C. L., Huang, B., Rodgers, W., \& Tice, T. N. (2007). Psychosocial mediation of religious coping styles: A study of short-term psychological distress following cardiac surgery. Personality and Social Psychology Bulletin, 33(6), 867-882. doi: $10.1177 / 0146167207301008$

Bandura, A. (1977). Self-efficacy: Toward a unifying theory of behavioral change. Psychological Review, 84(2), 191-215. doi: 10.1037/0033-295X.84.2.191

Beck, A. T., Ward, C. H., Mendelson, M. M., Mock, J. J., \& Erbaugh, J. J. (1961). An inventory for measuring depression. Archives of General Psychiatry,4(6), 561-571. doi: 10.1001/archpsyc.1961.01710120031004

*Black, J. L., Allison, T. G., Williams, D. E., Rummans, T. A., \& Gau, G. T. (1998). Effect of intervention for psychological distress on rehospitalization rates in cardiac rehabilitation patients. Psychosomatics, 39(2), 134-143. doi: 10.1016/S00333182(98)71360-X

Bostwick, J. M., \& Sola, C. L. (2007). An updated review of implantable cardioverter/ defibrillators, induced anxiety, and quality of life. Psychiatric Clinics of North America, 30(4), 677-688. doi:10.1016/j.psc.2007.07.002

*Brown, M. A., Munford, A. M., \& Munford, P. R. (1993). Behavior therapy of psychological distress in patients after myocardial infarction or coronary bypass. Journal of Cardiopulmonary Rehabilitation, 13(3), 201-210.

*Colella, T. J. F. (2009). The effect of a professionally-guided telephone peer support intervention on early recovery outcomes in men following coronary artery bypass graft surgery. Dissertation Abstracts International: Section B: The Sciences and Engineering, 70(7-B). 
*Dao, T. K., Youssef, N. A., Armsworth, M., Wear, E., Papathopoulos, K. N., \& Gopaldas, R. (2011). Randomized controlled trial of brief cognitive behavioral intervention for depression and anxiety symptoms preoperatively in patients undergoing coronary artery bypass graft surgery. Journal of Thoracic and Cardiovascular Surgery, 142(3), e109-e115. doi:10.1016/j.jtcvs.2011.02.046

Denollet, J., Schiffer, A. A., \& Spek, V. (2010). A general propensity to psychological distress affects cardiovascular outcomes: Evidence from research on the Type D (distressed) personality profile. Circulation: Cardiovascular Quality and Outcomes, 3(5), 546 - 557. doi:10.1161/CIRCOUTCOMES.109.934406

DerSimonian, R., \& Laird, N. (1986). Meta-analysis in clinical trials. Controlled Clinical Trials, 7(3), 177.

*Doering, L. V., Cross, R., Vredevoe, D., Martinez-Maza, O., \& Cowan, M. J. (2007). Infection, depression, and immunity in women after coronary artery bypass: A pilot study of cognitive behavioral therapy. Alternative Therapies in Health and Medicine, 13(3), 18-21.

*Doering, L. V., Chen, B., Cross, R., Magsarili, M. C., Nyamathi, A., \& Irwin, M. R. (2013). Early Cognitive Behavioral Therapy for Depression after Cardiac Surgery. The Journal of Cardiovascular Nursing, 28(4), 370-379. doi:10.1097/JCN.0b013e31824d967d

*Dougherty, C. M., Lewis, F. M., Thompson, E. A., Baer, J. D., \& Kim, W. Short-term efficacy of a telephone intervention by expert nurses after an implantable cardioverter defibrillator. Pacing and Clinical Electrophysiology, 27(12), 1594-1602. doi: 10.1111/j.1540-8159.2004.00691.x

*Dunbar, S. B., Langberg, J. J., Reilly, C. M., Viswanathan, B., McCarty, F., Culler, S. D., . . . Weintraub, W. S. (2009). Effect of a psychoeducational intervention on depression, anxiety, and health resource use in implantable cardioverter defibrillator patients. 
Pacing and Clinical Electrophysiology, 32(10), 1259-1271. doi: 10.1111/j.15408159.2009.02495.x

Dusseldorp, E., van Elderen, T., Maes, S., Meulman, J., \& Kraaij, V. (1999). A meta-analysis of psychoeduational programs for coronary heart disease patients. Health Psychology, 18(5), 506-519. doi: 10.1037/0278-6133.18.5.506

Egger, M., Davey Smith, G., Schneider, M., \& Minder, C. (1997). Bias in meta-analysis detected by a simple, graphical test. British Medical Journal, 315(7109), 629-634.

Egger, M., Jüni, P., Bartlett, C., \& for the, C. G. (2001). Value of flow diagrams in reports of randomized controlled trials. Journal of the American Medical Association, 285(15), 1996-1999. doi: 10.1001/jama.285.15.1996

*Freedland, K. E., Skala, J. A., Carney, R. M., Rubin, E. H., Lustman, P. J., Davila-Roman, V. G., . . Hogue Jr, C. W. (2009). Treatment of depression after coronary artery bypass surgery: A randomized controlled trial. Archives of General Psychiatry, 66(4), 387-396. doi:10.1001/archgenpsychiatry.2009.7

*Furze, G., Dumville, J. C., Miles, J. N., Irvine, K., Thompson, D. R., \& Lewin, R. J. (2009). "Prehabilitation" prior to CABG surgery improves physical functioning and depression. International Journal of Cardiology, 132(1), 51-58. doi: 10.1016/j.ijcard.2008.06.001

*Gallagher, R., McKinley, S., \& Dracup, K. (2003). Effects of a telephone counseling intervention on psychosocial adjustment in women following a cardiac event. Heart and Lung, 32(2), 79-87. doi: 10.1067/mhl.2003.19

*Habibovic, M., Denollet, J., Cuijpers, P., Spek, V., van den Broek, K. Warmerdam, L.,. . . Pedersen, S. (2014). E-health to manage distress in patients with an implantable cardioverter-defibrillator: Primary results of the WEBCARE trial. Psychosomatic Medicine, 76(8), 593-602. doi: 10.1097/PSY.0000000000000096 
Hagger, M. S., \& Chatzisarantis, N. L. D. (2014). It is premature to regard the ego-depletion effect as "Too Incredible". Frontiers in Psychology, 5, 298. doi: 10.3389/fpsyg.2014.00298

Hanssen, T. A., Nordrehaug, J. E., Eide, G. E., Bjelland, I., \& Rokne, B. (2009). Anxiety and depression after acute myocardial infarction: An 18-month follow-up study with repeated measures and comparison with a reference population. European Journal of Cardiovascular Prevention and Rehabilitation, 16(6), 651-659. doi: 10.1097/HJR.0b013e32832e4206

Hartling, L., Hamm, M., Klassen, T., Chan, A.-W., Meremikwu, M., Moyer, V., . . Offringa, M. (2012). Standard 2: Containing Risk of Bias. Pediatrics, 129(Supplement 3), S124S131. doi: 10.1542/peds.2012-0055E

Hawkes, A., \& Mortensen, O. (2006). Up to one third of individual cardiac patients have a decline in quality of life post-intervention. Scandinavian Cardiovascular Journal, 40(4), 214-218. doi: 10.1080/14017430600784343

Hedges, L. V., \& Olkin, I. (1985). Statistical methods for meta-analysis. Orlando, FL: Academic Press INC.

*Hermele, S. L. (2007). The effectiveness of a guided imagery intervention for patients undergoing coronary artery bypass graft surgery. Dissertation Abstracts International: Section B: The Sciences and Engineering, 67(10-B).

Hempel, S., Miles, J., Booth, M., Wang, Z., Morton, S., Shekelle, P., . . Shekelle, P. G. (2013). Risk of bias: A simulation study of power to detect study-level moderator effects in meta-analysis. Systematic Reviews, 2(1), 107.

Higgins, J., \& Green, S. (2008). Cochrane handbook for systematic reviews of interventions. England: John Wiley \& Sons Ltd.

Higgins, J. P. T., \& Thompson, S. G. (2002). Quantifying heterogeneity in a meta-analysis. 
Statistics in Medicine, 21(11), 1539. doi:10.1002/sim.1186

Höfer, S., Benzer, W., Alber, H., Ruttmann, E., Kopp, M., Schussler, G., \& Doering, S. (2005). Determinants of health-related quality of life in coronary artery disease patients: A prospective study generating a structural equation model. Psychosomatics, 46(3), 212-223. doi: 10.1097/01.hjr.0000201514.51461.64

Horodyska, K., Luszczynska, A., van den Berg, M., Hendriksen, M., Roos, G., De Bourdeaudhuij, I., \& Brug, J. (2015). Good practice characteristics of diet and physical activity interventions and policies: An umbrella review. BMC Public Health, 15(1), 19.

Hoyert, D. L., \& Xu, J. (2012). Deaths: Preliminary data for 2011. National Vital Statistics Report, 61(6), 1-7.

*Kohn, C. S., Petrucci, R. J., Baessler, C., Soto, D. M., \& Movsowitz, C. (2000). The Effect of psychological intervention on patients' long-term adjustment to the ICD: A prospective study. Pacing and Clinical Electrophysiology, 23(4), 450-456. doi: 10.1111/j.1540-8159.2000.tb00826.x

*Kuhl, E. A. (2007). Patient assisted computerized education for recipients of implantable cardioverter defibrillators (PACER): A randomized controlled trial of the PACER program. Dissertation Abstracts International: Section B: The Sciences and Engineering, 68(9-B).

*Lewin, R. J., Coulton, S., Frizelle, D. J., Kaye, G., Cox, H. (2009). A brief cognitive behavioural preimplantation and rehabilitation programme for patients receiving an implantable cardioverter-defibrillator improves physical health and reduces psychological morbidity and unplanned readmissions. Heart, 95 (1), 63-69. doi: doi: $10.1136 /$ hrt.2007.129890

*Lie, I., Arnesen, H., Sandvik, L., Hamilton, G., \& Bunch, E. H. (2007). Effects of a homebased intervention program on anxiety and depression 6 months after coronary artery 
bypass grafting: A randomized controlled trial. Journal of Psychosomatic Research, 62(4), 411-418. doi:10.1016/j.jpsychores.2006.11.010

Linden, W., Phillips, M. J., \& Leclerc, J. (2007). Psychological treatment of cardiac patients: a meta-analysis. European Heart Journal, 28(24), 2972-2984. doi: 10.1093/eurheartj/ehm504

Lindquist, R., Wyman, J. F., Talley, K. M., Findorff, M. J., \& Gross, C. R. (2007). Design of control-group conditions in clinical trials of behavioral interventions. Journal of Nursing Scholarship, 39(3), 214-221. doi: 10.1111/j.1547-5069.2007.00171.x

Lipsey, M. W., \& Wilson, D. B. (2001). Practical meta-analysis (Vol. 49). Thousand Oaks, CA: SAGE Publications.

Michie, S. (2008). Designing and implementing behaviour change interventions to improve population health. Journal of Health Services Research \& Policy, 13, 64-69.

Mirowsky, J., \& Ross, C. E. (2002). Selecting outcomes for the sociology of mental health: Issues of measurement and dimensionality. Journal of Health and Social Behavior, 43, $152-170$.

Moher, D., Liberati, A., Tetzlaff, J., Altman, D. G., \& Group, P. (2009). Preferred reporting items for systematic reviews and meta-analyses: The PRISMA statement. Journal of Clinical Epidemiology, 62(10), 1006-1012. doi: 10.1016/j.jclinepi.2009.06.005

Moher, D., Schulz, K. F., \& Altman, D. G. (2001). The CONSORT statement: Revised recommendations for improving the quality of reports of parallel-group randomised trials. The Lancet, 357(9263), 1191-1194. doi: 10.1016/S0140-6736(00)04337-3

Mullins, M. M., DeLuca, J. B., Crepaz, N., \& Lyles, C. M. (2014). Reporting quality of search methods in systematic reviews of HIV behavioral interventions (2000-2010): Are the searches clearly explained, systematic and reproducible? Research Synthesis 
Methods, 5(2), 116-130. doi: 10.1002/jrsm.1098

Norman, G., \& Streiner, D. (1993). Biostatistics: The bare essentials. St Louis: CV Mosby. Pedersen, S. S., \& Denollet, J. (2006). Is Type D personality here to stay? Emerging evidence across cardiovascular disease patient groups. Pacing and Clinical Electrophysiology, 30(12), 1546-1554. doi: 10.1111/j.1540-8159.2007.00905.x

*Qintar, M., George, J. J., Panko, M., Bea, S., Broer, K. A., Julie St. John, J. S.,... Chung, M. K. (2015). A prospective study of anxiety in ICD patients with a pilot randomized controlled trial of cognitive behavioral therapy for patients with moderate to severe anxiety. Journal of Interventional Cardiac Electrophysiology. doi: 10.1007/s10840015-9990-7

Rees, K., Bennett, P., West, R., Davey, S. G., \& Ebrahim, S. (2004). Psychological interventions for coronary heart disease. Cochrane Database of Systematic Reviews (2), CD002902. doi: 10.1002/14651858.CD002902.pub3

*Roncella, A., Pristipino, C., Cianfrocca, C., Scorza, S., Vincenzo Pasceri, V., Pelliccia, F...Speciale, G. (2013). One-year results of the randomized, controlled, short-term psychotherapy in acute myocardial infarction (STEP-IN-AMI) trial. International Journal of Cardiology, 170(2), 132-139. doi:10.1016/j.ijcard.2013.08.094

Salmoirago-Blotcher, E., \& Ockene, I. S. (2009). Methodological limitations of psychosocial interventions in patients with an implantable cardioverter-defibrillator (ICD): A systematic review. BMC Cardiovascular Disorders, 9(56). doi:10.1186/1471-2261-956

*Sears, S. F., Sowell, L. D., Kuhl, E. A., Kovacs, A. H., Serber, E. R., Handberg, E., . . Conti, J. B. (2007). The ICD shock and stress management program: A randomized trial of psychosocial treatment to optimize quality of life in ICD patients. Pacing and Clinical Electrophysiology, 30(7), 858-864. doi: 10.1111/j.1540-8159.2007.00773.x 
Shephard, R. J., \& Franklin, B. (2001). Changes in the quality of life: A major goal of cardiac rehabilitation. Journal of Cardiopulmonary Rehabilitation, 21(4), 189-200.

Škodová, Z., van Dijk, J. P., Nagyova, I., Rosenberger, J., Ondusova, D., Middel, B., \& Reijneveld, S. A. (2009). Psychosocial predictors of change in quality of life in patients after coronary interventions. Heart and Lung, 40(4), 331-339. doi: 10.1016/j.hrtlng.2009.12.007

Sola, C. L., \& Bostwick, J. M. (2005). Implantable Cardioverter-Defibrillators, Induced Anxiety, and Quality of Life. Mayo Clinic Proceedings, 80(2), 232. doi: $10.4065 / 80.2 .232$

*Sorlie, T., Busund, R., Sexton, J., Sexton, H., \& Sorlie, D. (2007). Video information combined with individualized information sessions: Effects upon emotional wellbeing following coronary artery bypass surgery: A randomized trial. Patient Education and Counseling, 65(2), 180-188. doi: 10.1016/j.pec.2006.07.006

Speilberger, C., Gorsuch, R., Lushene, R., Vagg, P., \& Jacobs, G. (1983). Manualfor the State-Trait Anxiety Inventory. Palo Alto, CA: Consulting Psychologists Press.

*Stein, T. R., Olivo, E. L., Grand, S. H., Namerow, P. B., Costa, J., \& Oz, M. C. (2010). A pilot study to assess the effects of a guided imagery audiotape intervention on psychological outcomes in patients undergoing coronary artery bypass graft surgery. Holistic Nursing Practice, 24(4), 213-222. doi: 10.1097/HNP.0b013e3181e90303

Thombs, B. D., Bass, E. B., Ford, D. E., Stewart, K. J., Tsilidis, K. K., Patel, U., . . Ziegelstein, R. C. (2006). Prevalence of Depression in Survivors of Acute Myocardial Infarction. Journal of General Internal Medicine, 21(1), 30-38. doi: 10.1111/j.15251497.2005.00269.x

Thompson, D. R., \& Ski, C. F. (2013). Psychosocial interventions in cardiovascular disease what are they? European Journal of Preventive Cardiology, 20(6), 916-917. doi: 


\section{$10.1177 / 2047487313494031$}

*Trzcieniecka-Green, A. \& Steptoe, A. (1996). The effects of stress management on the quality of life of patients following acute myocardial infarction or coronary bypass surgery. European Heart Journal, 17(11), 1663-1670.

Tully, P. J., \& Baker, R. A. (2012). Depression, anxiety, and cardiac morbidity outcomes after coronary artery bypass surgery: A contemporary and practical review. Journal of Geriatric Cardiology, 9, 197-208. doi: 10.3724/SP.J.1263.2011.12221

van Dixhoorn, J., \& White, A. (2005). Relaxation therapy for rehabilitation and prevention in ischaemic heart disease: A systematic review and meta-analysis. European Journal of Cardiovascular Prevention \& Rehabilitation, 12(3), 193-202. doi: 10.1097/01.hjr.0000166451.38593.de

Welton, N. J., Caldwell, D. M., Adamopoulos, E., \& Vedhara, K. (2009). Mixed treatment comparison meta-analysis of complex interventions: Psychological interventions in coronary heart disease. American Journal of Epidemiology, 169(9), 1158-1165. doi: 10.1093/aje/kwp014

Whalley, B., Rees, K., Davies, P., Bennett, P., Ebrahim, S., Liu, Z., . . Taylor, R. S. (2011). Psychological interventions for coronary heart disease. Cochrane Database of Systematic Reviews(8), CD002902. doi: 10.1002/14651858.CD002902.pub3

Whalley, B., Thompson, D., \& Taylor, R. (2014). Psychological interventions for coronary heart disease: Cochrane systematic review and meta-analysis. International Journal of Behavioral Medicine, 21(1), 109-121. doi: 10.1007/s12529-012-9282-x

World Health Organisation. (2011). Global status report on noncommunicable diseases 2010. from http://www.who.int/nmh/publications/ncd_report_full_en.pdf [Accessed: 22 August 2013]

Zigmond, A. S., \& Snaith, R. P. (1983). The hospital anxiety and depression scale. Acta 
Psychiatrica Scandinavica, 67(6), 361-370. doi:10.1111/j.1600-0447.1983.tb09716.x 